\title{
Functional Analysis of Cardiac MR Images using SPHARM Modeling
}

\author{
Heng Huang ${ }^{a}$, Li Shen ${ }^{b}$, James Ford $^{a}$, Fillia Makedon ${ }^{a}$, Rong Zhang ${ }^{c}$ \\ Ling $\mathrm{Gao}^{d}$, Justin Pearlman ${ }^{d}$ \\ ${ }^{a}$ DEVLAB, Computer Science Department, Dartmouth College, Hanover, NH, USA; \\ ${ }^{b}$ Computer and Information Science Department, University of Mass. Dartmouth, MA, USA; \\ ${ }^{c}$ Department of Automation, Shanghai Jiao Tong University, Shanghai, China; \\ ${ }^{d}$ Departments of Medicine \& Radiology, Division of Cardiology, Dartmouth Medical School, \\ Lebanon, NH, USA
}

\begin{abstract}
Visualization and quantification of cardiac function can provide direct and reliable indicators of cardiac health. The heart's operation occurs in three dimensions, and is dependent on three dimensional forces and ventricular geometry, making the observation of its shape important. Many approaches have been presented to extract cardiac shape and do functional analysis from a variety of imaging modalities. We apply a spherical harmonics (SPHARM) model to cardiac function analysis using magnetic resonance (MR) images. Our three dimensional SPHARM approach increases measurement accuracy over two dimensional approaches and also simplifies the management and indexing of clinical data by providing access to many important functional measures directly from the SPHARM representation.
\end{abstract}

Keywords: Medical Image Analysis, Cardiac MRI, Computer-Aided Diagnosis

\section{INTRODUCTION}

Cardiovascular disease (CVD) is the most common cause of death in the United States. Almost one million Americans die of CVD each year, constituting $42 \%$ of all deaths. Accurate diagnosis is important for effective treatment of cardiac disease. Magnetic resonance imaging (MRI) provides a promising new method to study cardiac function. More accurate measures of heart function based on MRI could provide invaluable diagnostic information to clinicians on disease severity and long-term prognoses. Three dimensional (3D) model-based analysis of cardiac shape and motion has the potential to provide rich morphological and functional information, ${ }^{1}$ which are well appreciated and a subject of great interest both to cardiovascular scientists and clinical cardiologists. $^{2}$ The effort to provide accurate three dimensional modeling is very important for cardiac function measurements and analysis, since some small inaccuracies in the wall thickness measurements could be amplified into significant errors when such measurements are used to calculate wall thickening. A variety of three dimensional modeling techniques are now available for obtaining cardiac functional analysis. Frangi et al. provided a review $^{1}$ in which a large number of cardiac modeling methods are introduced and compared. Staib and Duncan use sinusoidal basis functions to approximate the endocardium (the lining of the interior surface of the heart chambers) of the left ventricle (LV). ${ }^{3}$ Bardinet et al. use an extended superquadrics technique to describe the left ventricle. ${ }^{4}$

This paper first provides a 3D model to accurately measure and visualize heart and left ventricular geometry and function. In addition, two special SPHARM descriptors are generated for left ventricular wall thickness and wall stress. Based on spatio-temporal MR images, our spherical harmonics modeling approaches are valuable research tools for understanding cardiac function and its relationship with stages of disease. At the same time,

Further author information: (Send correspondence to Heng Huang)

Heng Huang: E-mail: hh@cs.dartmouth.edu, Telephone: 16036461694

Li Shen: lshen@umassd.edu; James Ford: jford@cs.dartmouth.edu; Fillia Makedon: makedon@cs.dartmouth.edu

Ling Gao: ling.gao@dartmouth.edu; Justin Pearlman: justin.pearlman@dartmouth.edu 
because a large number of images are required in cardiac research, clinicians and scientists also need to expediently manage (generate, store, search, read, and even classify) 3D models and their functional parameters. Our models simplify management in combining many functional analyses together. A fused data set is potentially useful for further explaining the disease process.

\section{BACKGROUD}

In previous work, Chang et al. presented motion and shape modeling primitives for the left ventricle. ${ }^{5}$ Since they began from an initial radial surface function $r(\theta, \phi)$, the method was representing only star-shaped or convex objects without holes. However, the heart and its chambers are not actually star-shaped, especially as there are papillary muscles on the endocardium. We therefore adopt the SPHARM expansion technique ${ }^{6}$ to create shape descriptions for $3 \mathrm{D}$ heart surfaces, ${ }^{7}$ where we employ an equal area mapping approach to parameterize each vertex on a heart surface onto the surface of a unit sphere.

This section first describes the cardiac MR images that are used in our experiments. The following subsections then describe the SPHARM surface description for the heart and left ventricle.

\subsection{Materials}

Cardiac MRI captures 3D images of the heart during its normal operation, with acquisition timed according to heartbeat frequency so that a fixed number of images are acquired during each heartbeat. In this work imaging was performed on a 1.5 Tesla scanner (Genesis Signa, GE Medical systems) with flip angle $20^{\circ}$ and slice thickness of $5 \mathrm{~mm}$, producing sequences of heart images in DICOM format. Each sequence consists of 20 volume images that together represent one complete heartbeat cycle. We have two image data sets for study: one comes from patients' MRI, and the other from MRI of pig hearts.

\subsection{Spherical Harmonics (SPHARM) Surface Description}

Spherical harmonics functions have certain mathematical properties that make them attractive for surface modeling $^{8}$ : orthogonality, completeness, and ordering in spatial frequency. Since the heart and its chambers have the topology of a sphere, spherical harmonics descriptors are reasonable to use to describe their shapes.

An input object surface is assumed to be defined by a square surface parameter mesh converted from an isotropic voxel representation. Two steps are involved in converting the object surface to its SPHARM shape description: (1) surface parameterization, and (2) SPHARM expansion.

Surface parameterization aims to create a continuous and uniform mapping from the object surface to the surface of a unit sphere. The parameterization is formulated as a constrained optimization problem with the goals of preserving area and topology while minimizing distortions; $\operatorname{see}^{9}$ for details. The result is a mapping of two spherical coordinates $\theta$ and $\phi$ to each point $\mathbf{r}(\theta, \phi)$ on a surface:

$$
\mathbf{r}(\theta, \phi)=\left(\begin{array}{c}
x(\theta, \phi) \\
y(\theta, \phi) \\
z(\theta, \phi)
\end{array}\right)
$$

$\theta \in[0, \pi]$ is the polar angle and $\phi \in[0,2 \pi)$ is the azimuthal angle. When the free variables $\theta$ and $\phi$ range over the whole sphere, $\mathbf{r}(\theta, \phi)$ ranges over the whole object surface. SPHARM expansion is then used to expand the object surface into a complete set of SPHARM basis functions $Y_{l}^{m}$, where $Y_{l}^{m}$ denotes the spherical harmonic of degree $l$ and order $m$ ( see $^{9}$ for details). The expansion takes the following form:

$$
\mathbf{r}(\theta, \phi)=\sum_{l=0}^{\infty} \sum_{m=-l}^{l} \mathbf{c}_{l}^{m} Y_{l}^{m}(\theta, \phi),
$$

where

$$
\mathbf{c}_{l}^{m}=\left(\begin{array}{c}
c_{l x}^{m} \\
c_{l y}^{m} \\
c_{l z}^{m}
\end{array}\right),
$$




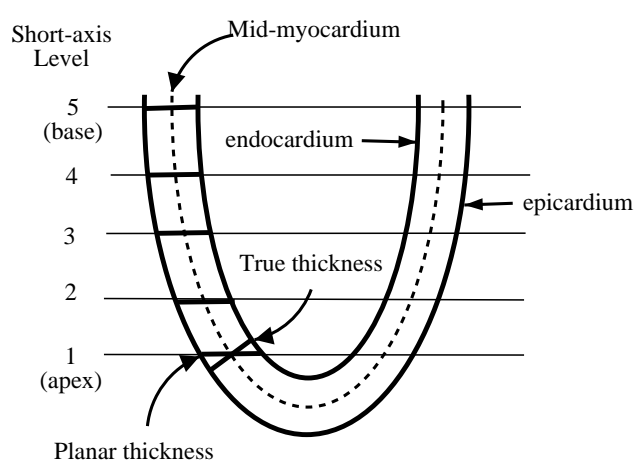

Figure 1. Planar wall thickness methods will overestimate the wall thickness.

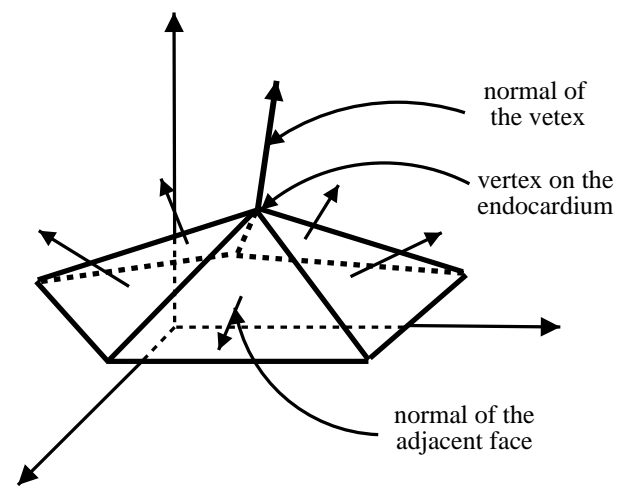

Figure 2. Normal vector $\vec{n}$ is the average of the outer normals $\overrightarrow{h_{i}}$ of the adjacent triangles.

The spherical harmonic basis functions are defined as:

$$
Y_{l}^{m}(\theta, \phi) \equiv \sqrt{\frac{2 l+1}{4 \pi} \frac{(l-m) !}{(l+m) !}} P_{l}^{m}(\cos \theta) e^{i m \phi},
$$

where $P_{l}^{m}(\cos \theta)$ are associated Legendre polynomials (with argument $\cos \theta$ ) that is defined by the differential equation

$$
P_{l}^{m}(x)=\frac{(-1)^{m}}{2^{l} l !}\left(1-x^{2}\right)^{m / 2} \frac{d^{m+l}}{d x^{m+l}}\left(x^{2}-1\right)^{l},
$$

where $l$ and $m$ are integers with $-l \leq m \leq l$.

The coefficients $\mathbf{c}_{l}^{m}$ are $3 \mathrm{D}$ vectors. Their components, $c_{l x}^{m}, c_{l y}^{m}$, and $c_{l z}^{m}$ are usually complex numbers. The coefficients up to a user-desired degree can be estimated by solving a set of linear equations in a least square fashion. The object surface can be reconstructed using these coefficients, and using more coefficients leads to a more detailed reconstruction. Thus, a set of coefficients actually form an object surface description.

During the heart cycle, the heart not only dilates and contracts, but also rotates within certain small angles. Given a set of shape sequences, we align all the shapes within the same sequence using a landmark-based method, where landmarks are manually defined by experts.

\section{APPLICATIONS AND RESULTS}

Currently LV volume, ejection fraction, wall thickness and wall stress are perhaps the most frequently used indexes of cardiac performance and patient prognosis. In practice, since 3D measurements can avoid geometric distortions, they are potentially more accurate than $2 \mathrm{D}$.

\subsection{Estimation and Analysis of Wall Thickness}

$\mathrm{LV}$ wall thickness has been linked to regional ischemia ${ }^{10}$ and dysfunctional contraction. ${ }^{11}$ However, many earlier studies for wall thickness measurements were made within a single imaging plane of short-axis MR images, and therefore overestimate true wall thickness. These errors are proportional to the curvature of the wall, and in Fig. 1 the difference between planar thickness and true thickness are obvious revealed. ${ }^{12}$ Recently many $3 \mathrm{D}$ approaches were developed to estimate the wall thickness when the wall curvature was taken into account. Buller et al. ${ }^{12}$ calculated the wall thickness using the formula $W T_{3 D}=W T_{2 D} \times \sin \alpha$, which can measure the wall thickness of points that are on slices. They calculate $\alpha$ using two planes, but the true $\alpha$ should be measured between two lines in 3-D space. Sometimes these two values of $\alpha$ are very different. Hubka et al. ${ }^{13}$ reconstructed the $3 \mathrm{D}$ model using 3-D echocardiography (3DE). But 3DE is not good at recovering the shape of the left ventricle. 


\begin{tabular}{lcccc}
\hline & Anterior(2D, 3D) & Septal $(2 \mathrm{D}, 3 \mathrm{D})$ & Lateral(2D,3D) & Inferior(2D, 3D) \\
\hline Low & $6.9,5.7$ & $5.0,4.4$ & $4.2,3.5$ & $6.0,5.6$ \\
Mid & $11.6,12.7$ & $8.2,8.4$ & $4.0,4.1$ & $13.1,14.3$ \\
High & $8.0,8.1$ & $5.0,5.0$ & $8.1,7.9$ & $5.0,5.1$ \\
\hline
\end{tabular}

Table 1. End-diastolic $2 \mathrm{D}$ and $3 \mathrm{D}$ wall thickness comparison in different direction of LV

MRI made it possible for researchers to improve the accuracy of shape measurements and visualization. Based on MRI the SPHARM descriptors help us to assess the global and regional variations of wall thickness rapidly and accurately.

In our approach, two 3D surfaces are constructed for the endocardium and epicardium of the LV through the SPHARM model. In Fig. 2, for each vertex $v_{\text {endo }}$ on the endocardial surface, let its normal vector $\vec{n}$ be the average of the outer normals $\overrightarrow{h_{i}}$ of the adjacent triangles that include this vertex. Transversing outwards to the epicardial surface, the normal vector passes through one triangle on the epicardial surface; let this point of intersection be denoted as $v_{e p i}$. The $3 \mathrm{D}$ wall thickness of $v_{\text {endo }}$ is defined as the distance between the vertex $v_{\text {endo }}$ and $v_{e p i}$.

Furthermore, we create the SPHARM descriptor for wall thickness using the current endocardial vertices, then the values of wall thickness, $\theta$, and $\phi$ are put into:

$$
\begin{gathered}
W T_{3 D}(\theta, \phi)=\sum_{l=0}^{\infty} \sum_{m=-l}^{l} c_{t_{l}}^{m} Y_{l}^{m}(\theta, \phi) \approx \sum_{l=0}^{L} \sum_{m=-l}^{l} c_{t_{l}}^{m} Y_{l}^{m}(\theta, \phi) \\
c_{t_{l}}^{m}=\left\langle W T_{3 D}(\theta, \phi), Y_{l}^{m}(\theta, \phi)\right\rangle=\int_{0}^{\pi} \int_{0}^{2 \pi} W T_{3 D}(\theta, \phi) Y_{l}^{m}(\theta, \phi) d \phi \sin \theta d \theta,
\end{gathered}
$$

The coefficients $c_{t_{l}}^{m}$ also can be obtained by solving a set of linear equations. $L$ is used to truncate the series of spherical harmonic coefficients to a finite number of terms. Thereafter, the wall thickness of each LV is represented by its own coefficients set $c_{t_{l}}^{m}$, and its wall thickness can be globally calculated from the wall thickness SPHARM descriptor. Because our measurement approaches aren't dependent on the cardiac geometry, we can measure the wall thickness of every region spreaded on the epicardium. For example, the method shown in Fig.1 cannot measure the wall thickness in the apex part of left ventricle, but our method can accurately measure them.

Table 1 lists planar and 3D wall thickness results in our experiments. We measure the planar (measured using ImageJ from MRI 2D slices) and 3D (by SPHARM model) of LV wall thickness from 20 pigs' MRI, and use their average measurements. Four normal directions are considered, and low section is close to apex of LV and mid section is close to papillary muscles plane and high section is close to the base of LV. In the low part, the planar wall thicknesses overestimate the wall thickness more, because the bias line is longer than the perpendicular line. The other big gaps appear in mid section of anterior and inferior direction, because the endocardium in our experiment doesn't include the papillary muscles.

Fig. 3 shows the observed wall thickness of the LV at the end-diastolic phase of a heartbeat cycle, with thickness represented by color. In this case, thinning of the apical (top) wall (that is created by myocardial infarction) is shown in the patient's heart. This visualization of LV thickness shows its extent - pathological section is distinctly displayed by the blue color of the apex. From this visual and numerical 3D information, a clinician could be assisted in making an accurate diagnosis and treatment plan.

\subsection{Regional Wall Stress Analysis}

Because myocardial (heart muscle) wall stress is closely related to both pathologic cardiac remodeling and ultimately to left ventricular decompensation, an accurate description of regional wall stress distribution may improve the ability of clinicians to identify problems in the absence of symptoms. ${ }^{14}$ Although global left ventricular wall stress is a useful clinical measure that has been correlated with many other parameters, potentially 




Figure 3. On the left, a visualization of a patients heart is shown, including right and left ventricle (represented by both endocardium and epicardium). In the right figure, back and front views of the left ventricle are shown with color indicating the wall thickness at the end-diastole phase. Coordinates are based on 2D MRI pixels and correspond to 1.49 $\mathrm{mm}$.

more significant cases involve detecting regional variations in myocardial wall stress that could indicate abnormalities. For example, regional end-systolic stress is helpful in the serial evaluation, optimal management, and early identification of left ventricular decompensation in patients with severe aortic insufficiency. ${ }^{14}$

When we calculate the wall thickness in subsection 3.1, we also select the midpoints of vectors between the endocardium and epicardium. By using these midpoints, we can reconstruct the mid-myocardium for LV, which is the center of the heart wall, as shown in Fig. 1. Because the wall stress is given by the equilibrium of forces between the LV cavity and the wall, researchers always use the mid-myocardium when calculating the wall stress of the LV.

End-systolic wall stress could be calculated by the Grossman formula ${ }^{15}$ :

$$
W S=0.133 \times S P \times \frac{R}{2 T \times\left(1+\frac{T}{2 R}\right)}
$$

where SP is the peak systolic ventricular blood pressure $(\mathrm{mmHg})$ that is used for calculating end-systolic pressure-volume relations, ${ }^{16}$ and 0.133 is a factor for converting $\mathrm{mmHg}$ to $10^{3} \mathrm{~N} / \mathrm{m}^{2} . R$ is the inner radius, which has often been estimated on 2D slices, and which we calculate in 3D by two steps. First, the centroid of the mid-myocardium is found by SPHARM modeling. Second, 3D inner radiuses are obtained by connecting every point on the mid-myocardium to the centroid. We still use the subsection 3.1 method to obtain the wall stress SPHARM descriptor of the LV. Fig. 4 shows the variation of end-systolic 3D wall stress for a pig's LV in four different directions. Regional variations in the wall stress are evident.

\subsection{Volume, Stroke Volume and Ejection Fraction}

$\mathrm{LV}$ volume is the basic parameter required to derive the other cardiac functional indexes, such as stroke volume and ejection fraction. Stroke volume $(S V)$ is the volume of blood ejected by a ventricle per heartbeat. The stroke volume is calculated as the difference between end-diastolic and end-systolic volumes:

$$
S V=\text { end-diastolic volume }(E D V) \text { - end-systolic volume }(E S V) \text {. }
$$




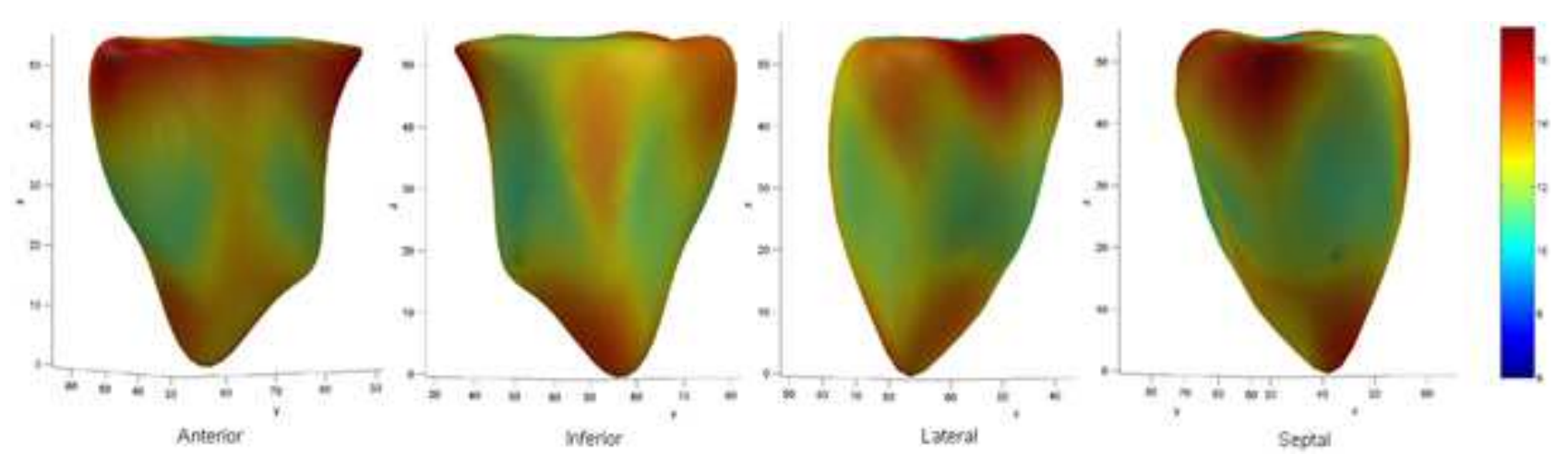

Figure 4. Variation of end-systolic 3D wall stress are visualized for mid-myocardium in four different directions of a pig heart using $150 \mathrm{mmHg}$ as the systolic blood pressure.

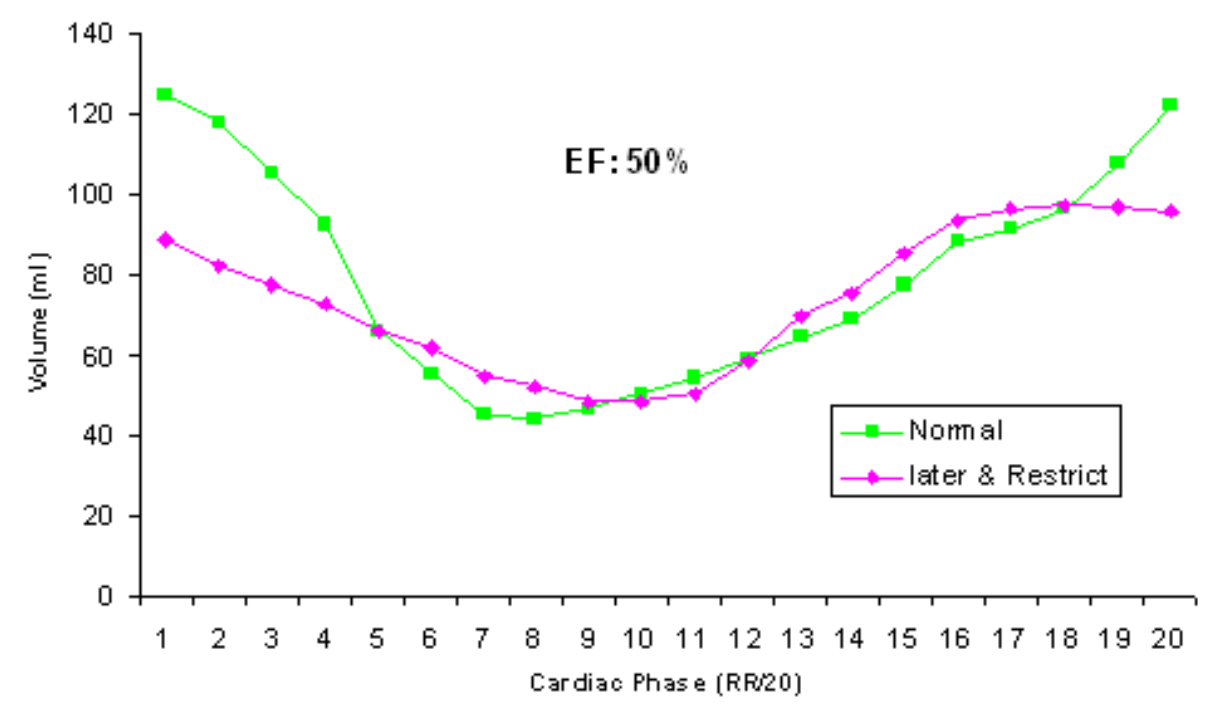

Figure 5. The variation in LV volume of the patient and healthy control during one heart cycle. $E F$ of patient is $50 \%$ and $S V_{\text {patient }}<S V_{\text {normal }}$

In our previous paper, ${ }^{7}$ the volume of the $\mathrm{LV}$ is calculated as the sum of the cavity areas multiplied by the section interval of MRI 2D slices (section thickness + section gap). Now a fast and more accurate method is implemented in this paper.

A point is picked inside the LV cavity, and it is connected with the vertices on the reconstructed 3D surface to form tetrahedra. This point should be parallel to the position of the papillary muscles on the $z$ axis and close to the centroid in the $x$ and $y$ axes, thereby reducing the measurement error introduced by the papillary muscles. The LV volume is then computed by summing the volumes of the tetrahedra.

Ejection fraction $(E F)$ is a global index of LV fiber shortening ${ }^{1}$ and the most commonly used parameter in the evaluation of LV pump function. It is determined by stroke volume:

$$
E F=\frac{S V}{E D V} \times 100 \%=\frac{E D V-E S V}{E D V} \times 100 \%
$$

In Fig. 5, the LV volume variation for one heart cycle as calculated by our SPHARM model are shown for the 
patient and healthy control. The plot shows that the patient's heart contracts to its end-systolic volume later than in healthy control, and that its end-diastolic volume is also restricted. $S V_{\text {patient }} \approx 43(\mathrm{ml})$ is far less than $S V_{\text {normal }} \approx 85(\mathrm{ml})$.

\subsection{Wall Thickening}

The other sensitive indicator of dysfunctional contraction is wall thickening. ${ }^{11}$ The finding of Azhari et al. has impelled many scientists to look for methods to quantify wall thickening. ${ }^{1}$ Three dimensional wall thickening $(3 D W T)$ is defined by end-systolic wall thickness $(E S T)$ and end-diastolic wall thickness $(E D T)^{15}$ :

$$
3 D W T(\%)=(E S T-E D T) / E D T .
$$

The calculation of global wall thickening can be determined directly from the coefficients of the wall thickness SPHARM descriptors that were introduced in subsection 3.1. Using Parseval's lemma, we can obtain:

$$
\begin{gathered}
E D T=\left(\frac{1}{4 \pi} \cdot \oint\|r(t)\|^{2} d t\right)^{1 / 2} \approx\left(\frac{1}{4 \pi} \cdot \sum_{l=0}^{L} \sum_{m=-l}^{l}\left|c_{t_{l}}^{m}\right|^{2}\right)^{1 / 2}, \\
E S T-E D T \approx\left(\frac{1}{4 \pi} \cdot \sum_{l=0}^{L} \sum_{m=-l}^{l}\left|c_{t_{l}, E S T}^{m}-c_{t_{l}, E D T}^{m}\right|^{2}\right)^{1 / 2} . \\
\text { Thus, } 3 D W T(\%) \approx\left(\sum_{l=0}^{L} \sum_{m=-l}^{l}\left|c_{t_{l}, E S T}^{m}-c_{t_{l}, E D T}^{m}\right|^{2} / \sum_{l=0}^{L} \sum_{m=-l}^{l}\left|c_{t_{l}}^{m}\right|^{2}\right)^{1 / 2} .
\end{gathered}
$$

This form of the 3DWT equation, which relates 3DWT to the SPHARM coefficients, allows a convenient calculation of wall thickening without access to the full MRI data.

\section{DISCUSSION}

We have presented novel cardiac functional analysis methods using SPHARM modeling approaches. Using these approaches, multiple components of 3D cardiac function can be quantified and studied based on a set of SPHARM descriptors. Because our shape models do not make any assumptions about the geometry of the left ventricle, analysis based on them should be very helpful for clinical applications and fundamental research.

Using SPHARM-based descriptors for wall thickness and stress makes it easier to manage patient information. For example, for every left ventricle, 20 sets of wall thickness SPHARM coefficients and their shape descriptors during one heart cycle are stored into database. After a researcher selects a pair of $\theta$ and $\phi$ or makes a landmark on the 3D surface using our software (that also transfers landmark into $\theta$ and $\phi$ ) for an interesting region of left ventricle, SPHARM coefficients sets are read for every left ventricle from a database. Using this information, wall thickness differences and accompanying visualizations for each ventricle can be calculated and rendered. Not having to store the original MRI data is very helpful for clinical and data classification research.

As a follow-up to these studies, more dynamic cardiac functional analysis will be included in our future work. We also plan to investigate using SPHARM-based descriptors in large-scale clinical databases as a means of supporting fast similarity searches and large-scale clinical comparisons.

\section{REFERENCES}

1. A. Frangi, W. Niessen, and M. Viergever, "Three-dimensional modeling for functional analysis of cardiac images: a review," IEEE Transactions on Medical Imaging 20(1), pp. 2-25, 2001.

2. M. A. O'Dell WG, "Imaging three-dimensional cardiac function," Annu Rev Biomed Eng. 2, pp. 431-456, 2000.

3. L. H. Staib and J. S. Duncan, "Model-based deformable surface finding for medical images," IEEE Transactions on Medical Imaging 15(5), pp. 720-731, 1996. 
4. E. Bardinet, L. D. Cohen, and N. Ayache, "A parametric deformable model to fit unstructured 3D data," Computer Vision and Image Understanding 71(1), pp. 39-54, 1998.

5. C. W. Chen, T. S. Huang, and M. Arrott, "Modeling, analysis, and visualization of left ventricle shape and motion by hierarchical decomposition," IEEE Transactions on Pattern Analysis and Machine Intelligence 16(4), pp. 342-356, 1994.

6. C. Brechbühler, G. Gerig, and O. Kübler., "Surface parametrization and shape description," Proc. Visualization in Biomedical Computing VBC'92, 1992.

7. H. Huang, L. Shen, F. Makedon, L. Gao, and J. Pearlman, "Three-dimensional analysis of cardiac magnetic resonance imaging using spherical harmonics model," appear in supplement to the Journal of the American College of Cardiology, March 7-10 2004.

8. G. Gerig, M. Styner, M. E. Shenton, and J. A. Lieberman, "Shape versus size: Improved understanding of the morphology of brain structures," in Proceedings of MICCAI01: Medical Image Computing and ComputerAssisted Intervention, 2001., pp. 24-31, 2001.

9. C. Brechbühler, G. Gerig, and O. Kübler., "Parametrization of closed surfaces for 3d shape description," Computer Vision and Image Understanding, 61(2):154-170, 1995.

10. G. Coppini, R. Poli, and G. Valli, "Recovery of the 3-D shape of the left ventricle from echocardiographic images," IEEE Transactions on Medical Imaging 14, pp. 301-317, 1995.

11. H. Azhari, S. Sideman, J. L. Weiss, E. P. Shapiro, M. L. Weisfeldt, W. L. Graves, W. J. Rogers, and R. Beyar, "Three-dimensional mapping of acute ischemic regions using MRI: wall thickening versus motion analysis," Am. J. Physiol. Heart Circ. Physiol. 259, pp. 1492-1503, 1990.

12. V. Buller, R. van der Geest, and J. R. M.D. Kool, "Accurate three-dimensional wall thickness measurement from multi-slice short-axis MR imaging," in Proc. IEEE Computers in Cardiology, pp. 245-248, (Los Alamitos, CA), 1994.

13. M. Hubka, J. Lipiecki, E. Bolson, R. Martin, B. Munt, S. Maza, and F. Sheehan, "Three-dimensional echocardiographic measurement of left ventricular wall thickness: In vitro and in vivo validation," $J A m$ Soc Echocardiogr 15(2), pp. 129-135, 2002.

14. B. P. Cupps, P. Moustakidis, B. J. Pomerantz, G. Vedala, R. P. Scheri, N. T. Kouchoukos, V. G. DavilaRoman, and M. K. Pasque, "Severe aortic insufficiency and normal systolic function: determining regional left ventricular wall stress by finite-element analysis," The Annals of Thoracic Surgery 76(3), pp. 668-675, 2003.

15. S. Delepine, A. P. Furber, F. Beygui, F. Prunier, P. Balzer, J.-J. L. Jeune, and P. Geslin, "3-D MRI assessment of regional left ventricular systolic wall stress in patients with reperfused MI," Am. J. Physiol. Heart Circ. Physiol. 284, pp. 1190-1197, 2003.

16. W. Grossman, E. Braunwald, T. Mann, L. McLaurin, and L. Green, "Contractile state of the left ventricle in man as evaluated from end-systolic pressure-volume relations," Circulation 56(5), pp. 845-852, 1977. 